\title{
Aplicación de tecnología ácida no corrosiva biodegradable para limpieza de pozos petroleros en Cuenca Oriente
}

\section{Application of biodegradable non-corrosive acid technology for cleaning oil wells in the eastern basin}

ARTÍCULO ORIGINAL

Recibido:

15/01/2019

Aprobado:

31/10/2019

\author{
Jaramillo Espinoza Carlos \\ (1) https://orcid.org/0000-0002-0130-8328 \\ Intermipetrol S.A \\ cjaramilloespinoza@intermipetrol.com
}

\author{
Cisneros Calderón Raúl \\ (1) https://orcid.org/0000-0002-2663-3426 \\ Intermipetrol S.A. \\ raulcis@intermipetrol.com
}

\section{RESUMEN}

En el presente artículo se demuestra la eficacia en la aplicación de nueva tecnología ácida no corrosiva biodegradable para la limpieza de pozos petroleros. Para su demostración se realizó ensayos de laboratorio sobre cupones metálicos con incrustaciones de carbonatos. Se tomaron dos cupones, uno sometido al efecto de ácido $\mathrm{HCl} 12 \%$ convencional mientras que el otro cupón al efecto de la tecnología de ácido no corrosivo biodegradable. De los resultados obtenidos se evidenció que el ácido no corrosivo biodegradable obtuvo mayor eliminación de carbonatos y menor desgaste del cupón metálico. Posterior se muestra una prueba de campo sobre un equipo con corrosión y carbonatos. La metodología aplicada consistió en sumergir el equipo de prueba en un recipiente en contacto con la tecnología ácida no corrosiva y se dejó en remojo por un lapso de 24 horas. Posterior se recuperó el equipo de prueba verificando por observación la limpieza de éste. Con el objetivo de verificar reacciones secundarias de la tecnología ácida no corrosiva biodegradable al contacto con crudo de la Cuenca Oriente del Ecuador, se realizó un ensayo de laboratorio de compatibilidades entre ambos fluidos observando separación de fases definida, buena tensión interfacial y poca o nula adsorción en las paredes de la botella de prueba y no hubo formación de emulsiones ni precipitados. Los resultados obtenidos del ensayo de compatibilidad concluyen la no presencia de reacciones secundarias al contacto con crudo. Por último, se muestra una gráfica de la historia de producción de un pozo de la Cuenca Oriente del Ecuador en la cual se señala el período de tiempo en donde se realizaron trabajos de limpieza en el pozo con ácido $\mathrm{HCl} 12 \%$ y ácido no corrosivo biodegradable. A partir de esta gráfica podemos deducir la caída y la recuperación de producción de crudo durante los dos eventos de limpieza ácida llevada a cabo. El histórico de producción muestra que con $\mathrm{HCl} 12 \%$ se obtuvo una caída de producción de 150 bppd mientras que con la tecnología ácida no corrosivo biodegradable la caída de producción fue de 80 bppd, por lo tanto, mayor producción en el segundo caso. Dentro de las conclusiones se pone de manifiesto que el uso de ácido no corrosivo biodegradable elimina las incrustaciones presentes en los pozos petroleros sin causar corrosión en los equipos mecánicos y con su propiedad biodegradable facilita la operación en campo.

\section{Palabras clave:}

Corrosión; carbonato; escala; incrustaciones; biodegradable; ácido; estimulación; limpieza de pozo.

\section{ABSTRACT}

This article demonstrates the effectiveness in the application of new non-corrosive biodegradable acid technology for oil well cleaning. For its demostration laboratory tests were carried out on metal coupons with carbonate inlays comparing with conventional acid. Two coupons were taken, one of them was subjected to the effect of conventional $12 \% \mathrm{HCl}$ acid while the other one to the effect of biodegradable non-corrosive acid technology. From the results obtained it was evidenced that the non-corrosive biodegradable obtained greater elimination of carbonates and less wear on the metallic coupon. The methodology applied was to immerse the test equipment in a container in contact with the noncorrosive acid technology and let it soak for a period of 24 hours. Subsequently, the test equipment was recovered by verifying the cleanliness of this by observation. In order to verify secondary reactions of biodegradable non-corrosive acid technology in contact with crude oil from the eastern basin of Ecuador, a laboratory compatibility test between both fluids was performed, observing defined phase separation, good interfacial tension and little or any 
adsorption on the walls of the test bottle and there was no formation of emulsions or precipitates. The results obtained from the compatibility test conclude the non-presence of secondary reactions to contact with oil. Finally, a graph of the production history of an oil well in the eastern basin of Ecuador is shown, in which it is pointed the period of time where cleaning jobs were carried out in the well with $12 \% \mathrm{HCl}$ acid and biodegradable non-corrosive acid. From this graph we can deduce the fall and recovery of crude oil production during the two acid cleaning events carried out. The graph shows a production drop of 150 obpd with $12 \% \mathrm{HCl}$ and a production drop of 80 obpd with the biodegradable non-corrosive acid technology, therefore, greater production in the second case. Within the conclusions it is shown that the use of biodegradable non-corrosive acid eliminates the scale present in the oil wells without causing corrosion in the mechanical equipment and with its biodegradable property facilitates field operation.

\section{Keywords:}

Corrosion; carbonate; scale; incrustations; biodegradable; acid; stimulation; well cleaning.

\section{INTRODUCCIÓN}

La industria hidrocarburífera realiza cambios constantes debido a que la operación se vuelve más compleja al incrementar diariamente el Bsw de los pozos. Esto conlleva a problemas en los pozos por varias razones, entre ellas obstrucciones debido a sólidos como por ejemplo: escala carbonatada, parafinas, asfaltenos, granos de arena, emulsiones, etc. De estas obstrucciones, la más común en los pozos petroleros presente en la Cuenca Oriente de la Amazonía ecuatoriana es el taponamiento por escala carbonatada en los punzados perforados, equipos BES, bombas HPS, líneas de superficie, facilidades, entre otros.

La limpieza de estos carbonatos comprende un procedimiento operacional con solventes y ácidos inorgánicos que si bien pueden tener efectividad representan otros inconvenientes que cada vez son más problemáticos en los procesos de la operadora . Para ofrecer una limpieza de pozo eficaz y eliminando los inconvenientes asociados al uso de químicos ácidos se ha desarrollado un nuevo esquema en base a la utilización de un ácido biodegradable y no corrosivo.

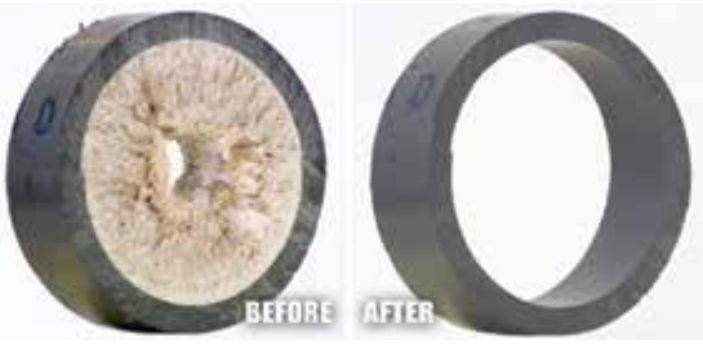

Figura 1: Muestra con escala (antes) y muestra sometida a limpieza ácida (después). Fuente: Apex Engineering. Products Corporation.
El presente artículo se basa en la utilización de tecnología ácida no corrosiva y biodegradable (Fig. 1) para su aplicación en limpieza de pozos petroleros ubicados en la Cuenca Oriente del Ecuador.

\section{METODOLOGÍA}

La metodología empleada para su aplicación es a través de la realización de ensayos de laboratorio con muestras metálicas, de crudo y de escala base carbonato en contacto con la tecnología ácida no corrosiva y evidenciar sus efectos. A nivel de campo se presenta el ejemplo de un caso del trabajo en un pozo petrolero y los efectos que se tubo en la producción del pozo.

Análisis de corrosión en acero y aleación níquel-cobre Se realizó un ensayo para corroborar si el producto cumple la propiedad de ser no corrosivo sobre piezas metálicas de acero y aleación cobre-níquel (CDA 706). Para ello, se elaboró el ensayo en condiciones específicas de $130^{\circ} \mathrm{F}$ con dos concentraciones ácidas, una al $100 \%$ y otra al $50 \%$.

Tabla 1. Resultados de ensayo de tasa de corrosión de ácido no corrosivo sobre cupones metálicos.

\begin{tabular}{|c|c|c|}
\hline & $\mathbf{1 0 0} \%$ ÁCIDO & $\mathbf{5 0} \% \mathbf{A}$ CIDO \\
\hline ACERO & 0.061 in/año & 0.041 in/año \\
\hline CDA 706 & 0.007 in/año & 0.007 in/año \\
\hline
\end{tabular}

Fuente: Calumet Associates, Inc.

De los resultados obtenidos de la tabla 1 , se comentó que no hay evidencia de fisuras, agujeros o algún tipo de desgaste en la superficie metálica.

\section{Ensayo de bio-degradabilidad del ácido}

De igual manera se realizó un ensayo sobre el ácido con el fin de determinar la propiedad de biodegradabilidad de este. Para ello se realizó un ensayo bioquímico de demanda de oxígeno (BOD) el cual es un indicador de biodegradabilidad. El ensayo fue realizado bajo las especificaciones técnicas de la última edición de la SMWW (Standard Methods for the Examination of Water and Wastewater), APHA (American Public Health Association), AWWA (American Water Works Association), WPCF (Water Pollution Control Facility).

Tabla 2. Resultado del ensayo BOD para la certificación de biodegradabilidad del ácido de prueba.

BOD $16 \mathrm{mg} / \mathrm{l}$

Fuente: ENVIRO-TEST, INC.

Con los resultados obtenidos de la tabla 2 , el producto ácido es considerado como biodegradable.

Ensayo de eliminación de incrustaciones vs. corrosión en cupones metálicos de pozo

Se realizó un ensayo de laboratorio con muestras de carbonatos presente en cupones metálicos al contacto con $\mathrm{HCl} 12 \%$ y tecnología ácida no 
corrosiva. Para cada ácido se realizó un ensayo por separado y se verificó el porcentaje de pérdida de peso por eliminación de carbonato en cada cupón. Como se observa en la figura 2, la eliminación de carbonatos fue mayor en el cupón con el ácido no corrosivo biodegradable con $90 \%$. Por otro lado, el cupón metálico con $\mathrm{HCl}(12 \%)$ obtuvo una pérdida de peso del $62 \%$ constatando mayor porcentaje en el primer ensayo.

Otro ensayo realizado fue la verificación del porcentaje de pérdida de peso en cupones metálicos limpios (sin escala) al ser sometidos por separado al contacto de $\mathrm{HCl} 12 \%$ y tecnología ácida no corrosiva (Fig. 3). Después de 4 horas de ensayo con el ácido no corrosivo biodegradable se obtiene una pérdida de peso de $0.04 \%$ mucho menor a su contraparte que obtuvo una pérdida de $0.37 \%$. En el mismo período de tiempo el cupón en contacto con $\mathrm{HCl}$ 12\% generó mayor desgaste del cupón metálico respecto al cupón de prueba con tecnología ácida no corrosiva.

\section{Prueba de campo sobre válvula de mariposa}

Se realizó un ensayo del ácido no corrosivo biodegradable en una válvula de mariposa con incrustaciones de escala y óxido. Se sumergió dicha válvula de mariposa con presencia de escala (Fig. 4) completa en un recipiente plástico con 5 gal de ácido no corrosivo biodegradable sin realizar ningún tipo de manipulación.

La reacción química entre escala y el ácido genera una espuma en poca cantidad que se deshace al ambiente. Al cabo de 20 minutos el ácido no corrosivo biodegradable ya había limpiado gran cantidad de escala y óxido presente en la muestra. Posterior, se dejó por un periodo de 24 horas en remojo.

Al cabo de las 24 horas se observó que la muestra no contenía ningún tipo de escala, incrustaciones u óxidos que tenía antes de realizar la prueba. Además, la muestra no mostró ningún tipo de desgaste en su cuerpo metálico, ni tampoco en el sello de caucho, ni en el cuerpo de la válvula.
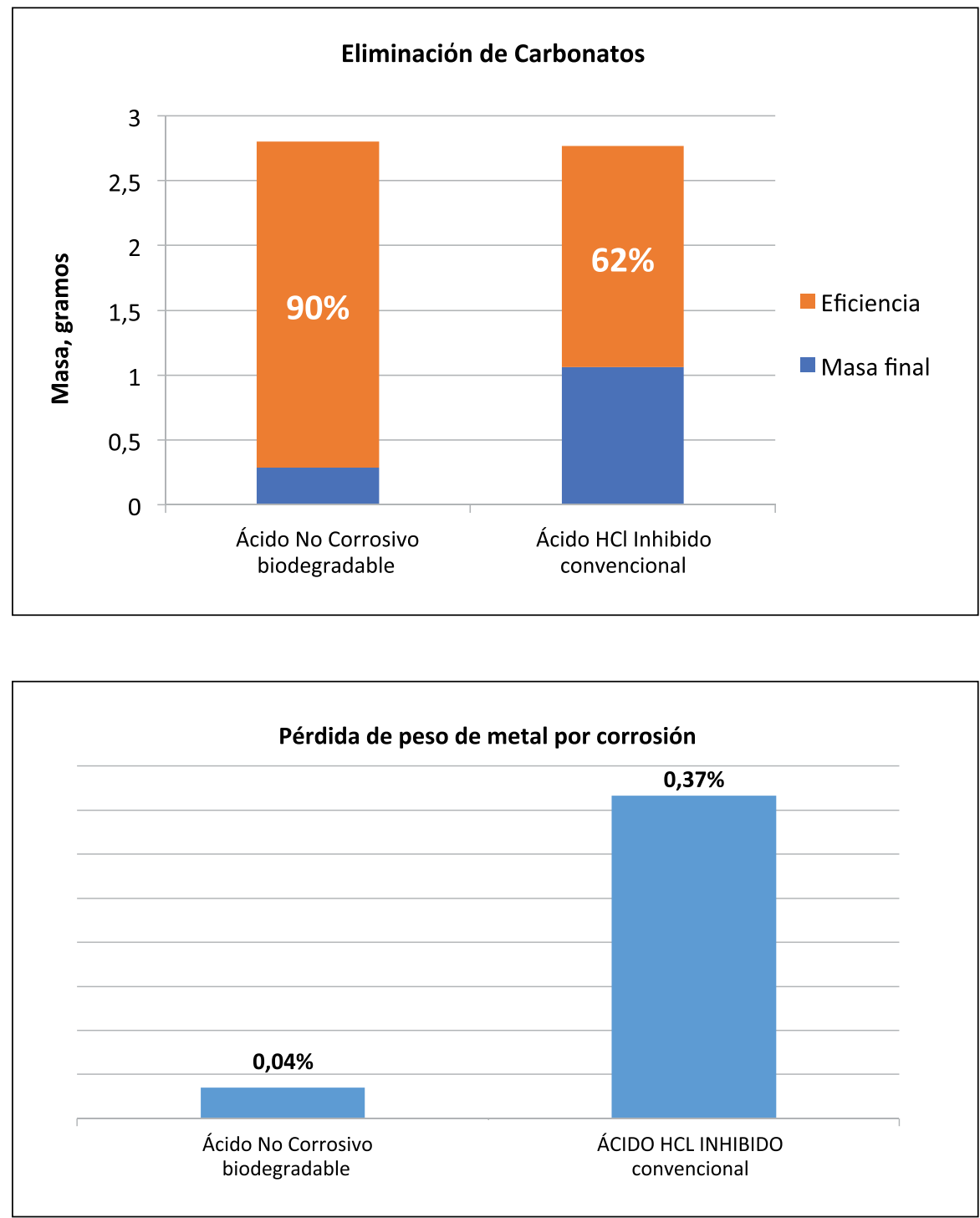

Figura 2: Comparativa de eliminación de incrustaciones de carbonatos. Tiempo 25 minutos. Fuente: Intermipetrol S.A.
Figura 3: Comparativa de pérdida de peso de metal por corrosión. Tiempo 4 horas.

Fuente: Intermipetrol S.A 


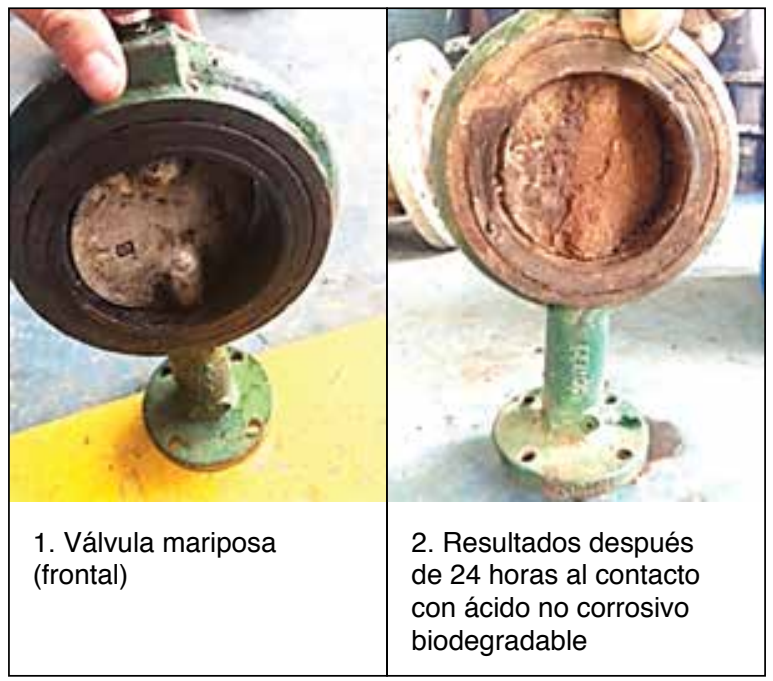

Figura 4: Comparativa del antes y el después de sumergir la válvula en ácido. Tiempo de contacto fue de 24 horas.

Fuente: Intermipetrol S.A.

\section{Ensayo con crudo Oriente de compatibilidad}

Se realizó un ensayo de compatibilidad con crudo de un pozo petrolero del Oriente ecuatoriano y el sistema ácido no corrosivo biodegradable (Fig. 5) con el objetivo de verificar si hay o no presencia de reacciones secundarias (emulsiones, precipitados) que pudiesen impedir la utilización de este ácido en pozos petroleros. El ácido no corrosivo fue preparado en mezcla con concentraciones específicas de surfactante y solvente mutual para tener una correcta separación de fases agua-aceite.

Del ensayo de compatibilidad realizado con crudo Oriente se puede apreciar acorde a la tabla 3, que al cabo de un minuto ya se contó con una separación de fases B (Oscura) y a los dos minutos ya se contó con una fase A (Definida). No se observó presencia de sedimentos, emulsiones, precipitados ni de lodo asfáltico.

Tabla No. 3: Resultados obtenidos del ensayo de compatibilidades con ácido no corrosivo biodegradable.

\begin{tabular}{|c|c|c|}
\hline & \multicolumn{2}{|c|}{ Muestra de crudo Oriente } \\
\hline $\begin{array}{c}\text { RELACIÓN } \\
\text { Crudo /Tto }\end{array}$ & $50 / 50$ & FA \\
\hline$\%$ & $100 \%$ & \\
\hline Tiempo 1 min & $100 \%$ & $\mathrm{~B}$ \\
\hline $\mathbf{2}$ & $100 \%$ & $\mathrm{~A}$ \\
\hline $\mathbf{5}$ & $100 \%$ & $\mathrm{~A}$ \\
\hline $\mathbf{1 0}$ & $100 \%$ & $\mathrm{~A}$ \\
\hline $\mathbf{2 0}$ & $100 \%$ & $\mathrm{~A}$ \\
\hline $\mathbf{3 0}$ & $100 \%$ & $\mathrm{~A}$ \\
\hline $\mathbf{6 0}$ & $0 \%$ & $\mathrm{~A}$ \\
\hline$\%$ Sludge & $*$ & \\
\hline Sedimentos & & \\
\hline
\end{tabular}

Elaborado por: Departamento de Petróleos, Energía y Contaminación (DPEC)-UCE.

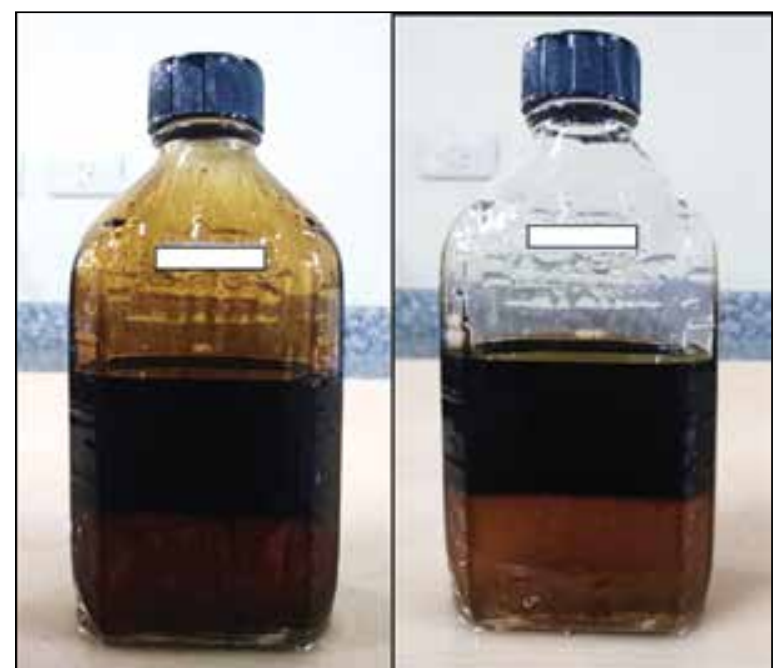

Figura 5: Ensayo de compatibilidades a 1 minuto y 30 minutos. Fuente: Departamento de Petróleos, Energía y Contaminación (DPEC)-UCE.

Ejecución del servicio en un pozo $\mathrm{x}$ del oriente ecuatoriano

En el pozo $X$ que se describe a continuación (Fig. 6), previo al 22 de noviembre que se observa en la gráfica, la operadora realizaba limpiezas ácidas con $\mathrm{HCl}$ convencional asistido con CTU. La operadora realizaba esta modalidad de limpieza en el pozo X cada 3 meses. Del histórico de producción se observa una caída de producción en el pozo de alrededor de 150 bppd. Posterior al 22 de noviembre, la operadora decide realizar trabajos de limpieza ácida con ácido no corrosivo biodegradable en modalidad bullheading. La metodología aplicada en el pozo fue de realizar el bombeo del sistema ácido no corrosivo biodegradable con camión de bombeo siguiendo una línea de flujo conectada directamente al cabezal del pozo y circulando el sistema ácido hasta desplazar el tratamiento a la profundidad donde se encontraba identificada la obstrucción sólida de carbonatos (escala) en el equipo de bombeo electrosumergible (BES).

Con el histórico de producción se observa una caída de 80 bppd (mucho menor a los 150 bppd de caída de producción que se obtuvo en el trabajo empleado con CTU y $\mathrm{HCl} 12 \%)$. La operadora obtuvo un incremento de producción en el pozo $X$ y las intervenciones en este pozo $X$ se prolongaron a periodos de 6 meses, de esta manera se redujo el número de intervenciones al pozo.

\section{DISCUSIÓN}

Acorde a los ensayos realizados y evidenciados se muestra que la tecnología ácida tiene propiedades de no corroer los metales y que es biodegradable. Adicional, los ensayos presentados también confirman estas aseveraciones en ensayos realizados en condiciones de campo. Contrastando los trabajos de limpiezas en pozos con $\mathrm{HCl} 12 \%$ y con el ácido no corrosivo biodegradable, en el segundo caso se obtienen ventajas como disminución de tiempo 


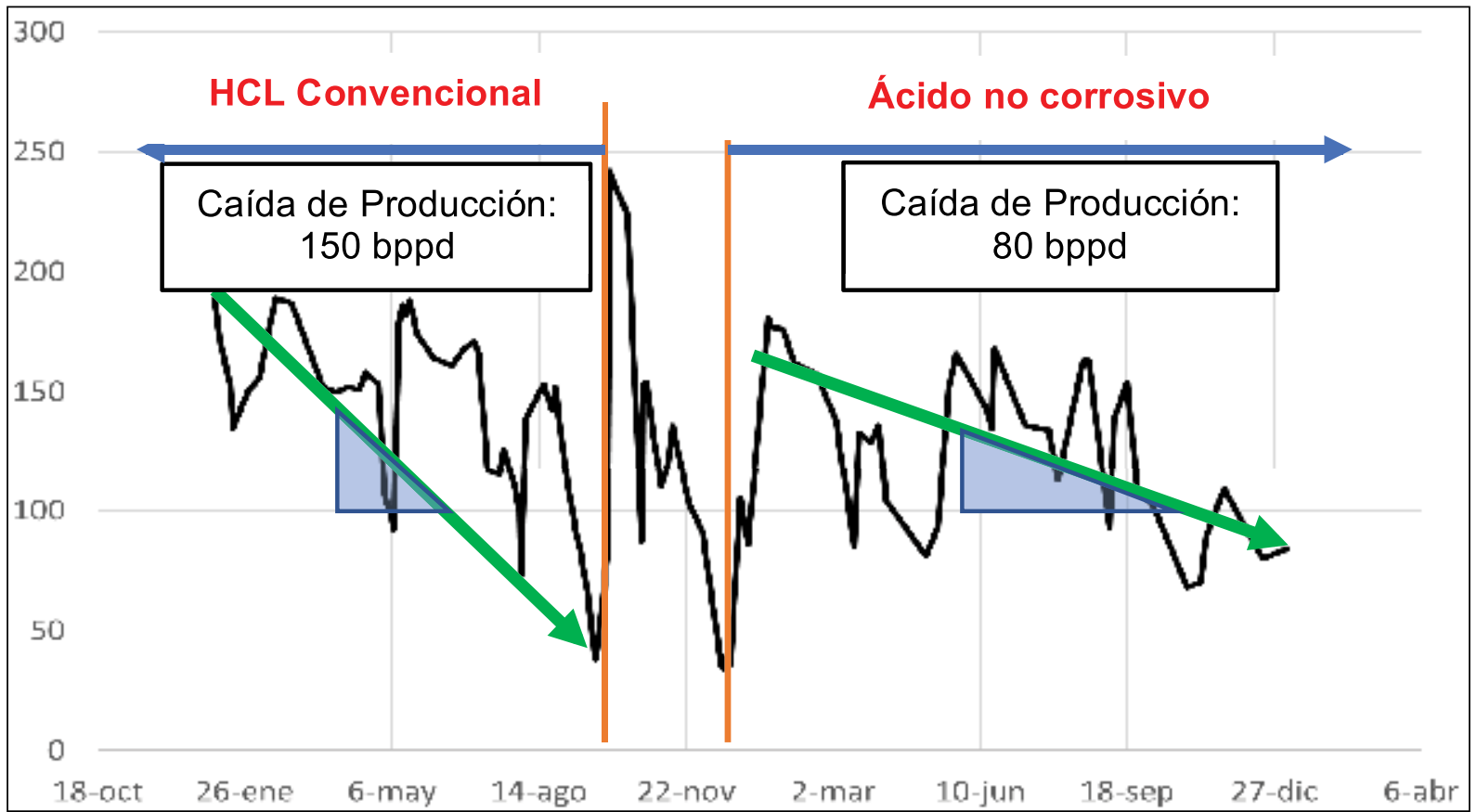

Figura 6: Histórico de producción del pozo X. Se aprecia el cambio en la caída de producción al implementar limpiezas ácidas con ácido no corrosivo biodegradable. Fuente: Intermipetrol S.A

de contacto sobre superficies metálicas, reducción del desgaste sobre equipos metálicos, efecto de desgaste puntualizado ya que reacciona al contacto con carbonatos.

Por otro lado, al ser un producto biodegradable da mayor confiabilidad en prolongar más aún el tiempo de exposición del ácido en el fondo del pozo. Este tipo de esquema de trabajo no se puede concebir al utilizar ácidos convencionales debido a que, a mayor tiempo de exposición, mayor corrosión y por ende problemas como fisuras por tanto va a incurrir en una limpieza incompleta de sólidos carbonatados. En contraste, al realizar trabajos con ácido no corrosivo biodegradable es posible disolver mayor cantidad de sólidos carbonatados dejando el ácido por un mayor tiempo de prolongación ya que el ácido no corrosivo biodegradable estará 100\% enfocado en la disolución de estos.

Por otro lado, comparando estos dos esquemas ácidos, especificamos que el ácido no corrosivo biodegradable no puede disolver sólidos que no correspondan a una base de carbonatos a diferencia de un ácido convencional que puede disolver otros tipos de sólidos presentes en el pozo.

\section{CONCLUSIONES}

Se determinó que el ácido no corrosivo biodegradable disuelve y elimina las incrustaciones de los equipos mecánicos con una gran eficacia y sin mayor esfuerzo en poco tiempo. El ácido no genera ningún tipo de emulsión al contacto con el crudo. La interfaz lineal que se forma en el ensayo de compatibilidades no muestra adsorción alguna lo que nos indica que el sistema ácido es compatible con otros aditivos y con el crudo pues no se produjo reacciones secundarias como emulsiones o precipitados.

De los resultados obtenidos de la comparación entre el ácido no corrosivo biodegradable y el $\mathrm{HCL}$ inhibido convencional se concluye que casi en el 30\% fue más efectivo el ácido no corrosivo biodegradable eliminando incrustaciones y $98 \%$ menos corrosivo que el HCL inhibido.

En base a los resultados determinados en el presente estudio tanto a nivel de laboratorio como su aplicación en campo podemos indicar que el ácido no corrosivo biodegradable facilita las operaciones de limpieza tanto en pozos como en equipos de superficie por varias razones; entre ellas su propiedad de biodegradación reduce procedimientos operacionales que suelen complicar a la operadora porque este producto no requiere procesos de post tratamientos debido a que se degrada con carbonatos; es un producto que no requiere regulación gubernamental, es amigable con el ambiente, con la salud y seguridad.

Adicional, las propiedades demostradas para la tecnología ácida no corrosiva biodegradable concluyen que el mencionado ácido de estudio únicamente actúa al contacto con sólidos a base de compuestos carbonatados como la escala presente en equipos de superficie y fondo de pozo; por lo tanto el producto puede estar en el pozo por un mayor tiempo prolongado sin causar ningún tipo de daño a los componentes mecánicos.

\section{REFERENCIAS}

- Apex Engineering Products Corporation (2018). Struvite Removal with RYDLYME. Recuperado de http://www.apexengineeringproducts.com/ 
struvite-removal/

- Chicago Spectro Service Laboratory, Inc. (2014). Recuperado de http://www.chicagospectro.com

- Corrales, E. (2013). Aplicación de nuevas tecnologías para optimizar la producción en el campo Palanda-Yuca Sur. (Tesis de pregrado). Universidad Central del Ecuador, FIGEMPA, Carrera de Ingeniería de Petróleos, Ecuador.

- Cruz, D. (2015). Análisis técnico de la implementación de estimulación matricial con unidad de coiled tubing para optimizar la producción del pozo PLAN-050H de la arena Hollín en la amazonia ecuatoriana. (Tesis de pregrado). Universidad Tecnológica Equinoccial, Facultad de Ciencias de la Ingeniería, Carrera de Ingeniería de Petróleos, Ecuador.

- Departamento de Petróleos, Energía y Contaminación. Facultad de Química. Universidad
Central del Ecuador. fig.secretaria.dpec@uce.edu.ec

- ENVIRO-TEST, Inc. (2018). Recuperado de http:// www.envirotestinc.com

- Gonzales, J., (2014). Daño a la formación en pozos petroleros. (Tesis de pregrado). Universidad Nacional Autónoma de México. Facultad de Ingeniería, México.

- INTERMIPETROL. (2015). RYDLYME in Ecuador(Oil Wells). Recuperado de http://www.intermipetrol. com/\#xl_xr_page_productos\%20biodegradables

- Leines, J. (2015). Optimización de la producción utilizando una técnica de fracturamiento alternativa en un pozo de un campo maduro de la cuenca oriente. (Tesis de pregrado). Universidad Central del Ecuador, FIGEMPA, Carrera de Ingeniería de Petróleos, Ecuador.

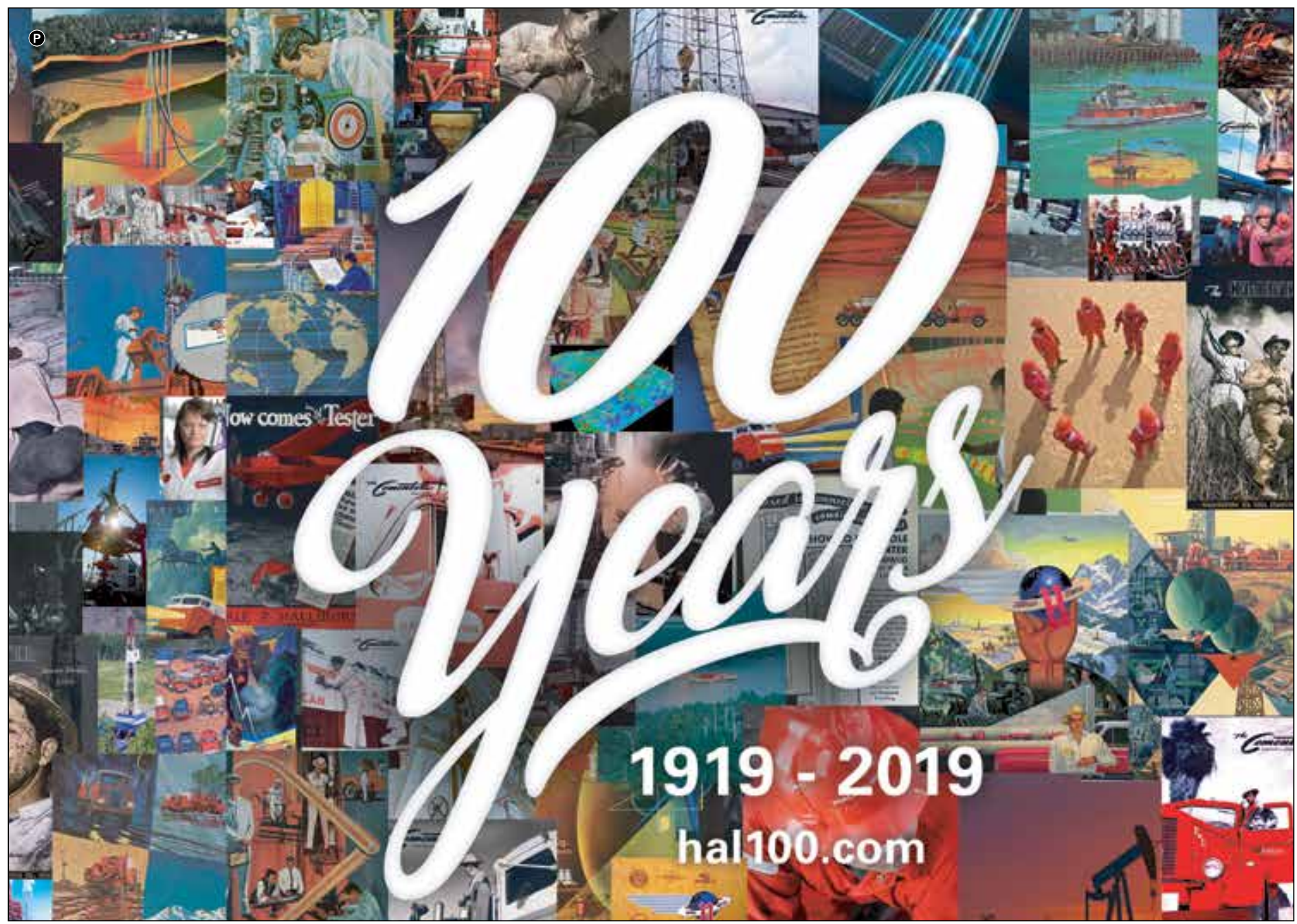

